\title{
Photochemical upconversion enhanced solar cells: Effect of a back reflector
}

Tim F. Schulze, ${ }^{1}$ Yuen Yap Cheng, ${ }^{1}$ Burkhard Fückel, ${ }^{1}$ Rowan W. MacQueen, ${ }^{1}$ Andrew Danos, ${ }^{1}$ Nathaniel J. L. K. Davis, ${ }^{1}$ Murad J. Y. Tayebjee, ${ }^{1}$

Tony Khoury, ${ }^{1}$ Raphaël G. C. R. Clady, ${ }^{1}$ N. J. Ekins-Daukes, ${ }^{2}$ Maxwell J. Crossley, ${ }^{1}$ Bernd Stannowski, ${ }^{3}$ Klaus Lips, ${ }^{4}$ and Timothy W. Schmidt ${ }^{1,5, *}$

${ }^{1}$ School of Chemistry, The University of Sydney, NSW 2006, Australia

${ }^{2}$ Department of Physics and the Grantham Institute for Climate Change, Imperial College, London, UK SW7 $2 A Z$

${ }^{3}$ Competence Centre Thin-Film- and Nanotechnology for Photovoltaics Berlin (PVcomB),

Helmholtz-Zentrum Berlin für Materialien und Energie, 12489 Berlin, Germany

${ }^{4}$ Institute for Silicon Photovoltaics, Helmholtz-Zentrum

Berlin für Materialien und Energie, 12489 Berlin, Germany

${ }^{5}$ Institute of Photonics and Optical Science,

The University of Sydney, NSW 2006, Australia

\begin{abstract}
Photochemical upconversion is applied to a hydrogenated amorphous silicon solar cell in the presence of a back-scattering layer. A custom-synthesized porphyrin was utilized as the sensitizer species, with rubrene as the emitter. Under a bias of 24 suns, a peak external quantum efficiency (EQE) enhancement of $\sim 2 \%$ was observed at a wavelength of $720 \mathrm{~nm}$. Without the scattering layer, the EQE enhancement was half this value, indicating that the effect of the back-scatterer is to double the efficacy of the upconverting device. The results represent a figure of merit of $3.5 \times 10^{-4} \mathrm{~mA} \mathrm{~cm}^{-2} \mathrm{sun}^{-2}$, which is the highest reported to date.
\end{abstract}

*t.schmidt@chem.usyd.edu.au 


\section{INTRODUCTION}

The energy consumption of our species is approximately $19 \mathrm{TW}$, and is projected to reach $21 \mathrm{TW}$ by 2020 . While this need is currently met by the burning of fossil fuels, concerns about climate change brought about by anthropogenic $\mathrm{CO}_{2}$ has inspired intense research into sustainable energy. While there are many possible alternative energy sources, more energy in the form of sunlight falls on our planet in one second than we require in a year. As such, solar photovoltaic energy holds much promise. Nevertheless, the roll-out of terawatts worth of solar cells requires an inexpensive manufacturing process utilizing abundant materials.[1, 2$]$

The first generation of solar cells is classed as those materials with efficiencies approaching the single threshold limit ( $\approx 30 \%$ for crystalline silicon).[3, 4] Indeed, crystalline silicon solar cells from UNSW have achieved in excess of 25\% energy conversion under standard illumination. [5] However, the cost of solar energy can be dramatically cut by reducing the cost of the cells, while maintaining reasonable energy conversion efficiency. The so-called second generation solar cells aim to achieve this.[2, 4] Second generation devices include thin-film silicon solar cells, and the photosynthesis-inspired "chemical" photovoltaic devices such as bulk-heteojunction and dye-sensitized solar cells.[6-8]

However, all of these solar cell designs are of the single-threshold type. A photovoltaic device with a single energy threshold is fundamentally limited to an energy conversion efficiency of $33.7 \%$ under the standard AM1.5G spectrum (1.34 eV threshold).[3, 9] This is due to two main losses: The energy in excess of the threshold is lost as heat, and subthreshold photons are not harvested by the device. The latter phenomenon accounts for the majority of lost solar energy in devices with thresholds exceeding about $1.4 \mathrm{eV}$.[10] Many second generation devices harvest light poorly above $800 \mathrm{~nm}(1.55 \mathrm{eV})$, and as such could be greatly improved if they could capture this lost sunlight, thus circumventing the singlethreshold limit to bring about a third generation photovoltaic device. $[4,11]$ One way to achieve this is by photochemical upconversion.[12]

In photochemical upconversion, light is harvested by sensitizer molecules which rapidly cross to a triplet state, temporarily storing the absorbed energy (see Fig. 1). By triplet energy transfer, the energy is passed to a second species, in excess - the emitter. The emitter triplet molecules encountering one another "annihilate" to bring about a single chromophore in an excited singlet state, poised to emit. Ensuing delayed fluorescence is at a shorter 


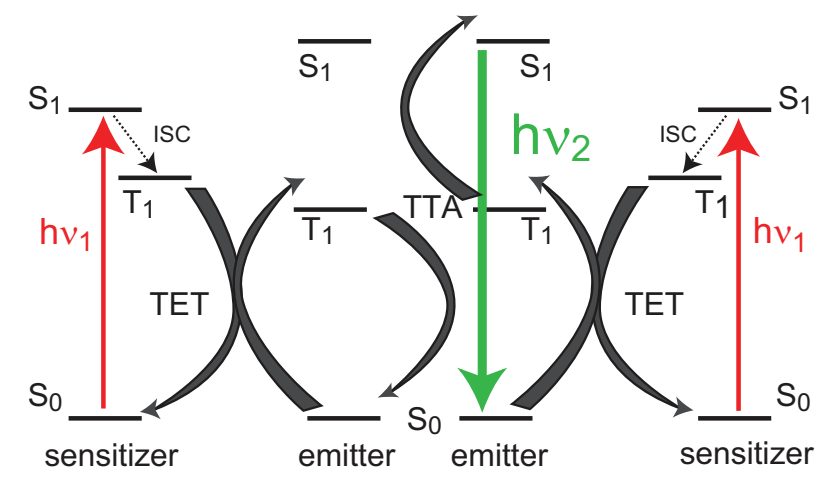

FIG. 1. A schematic of the TTA-UC process.

wavelength than the originally absorbed light. Since the energy is higher, this process is known as upconversion.

While triplet-triplet annihilation upconversion TTA-UC was thought to be fundamentally limited to an efficiency of $1 / 9$, on account of the probability of acquiring a singlet from two triplets,[13] we have shown this not to be the case.[14, 15] Indeed, we showed in our model system that in excess of $60 \%$ of triplets could be utilized in the TTA-UC process.[15] TTA$\mathrm{UC}$ is now seeing application in photochemical devices. It has been recently applied to drive water splitting in $\mathrm{WO}_{3}$ using sub-bandgap photons.[16] More recently, we demonstrated the first measurement of TTA-UC applied directly to hydrogenated amorphous silicon solar cells (a-Si:H).[17] In our work, we placed an upconverting mixture of porphyrin (as sensitizer) and rubrene (emitter) behind a a-Si:H cell. By taking the ratio of external quantum efficiency (EQE) curves with and without upconversion, we showed a peak relative increase in EQE of about $1 \%$ under the equivalent of 50 suns, amounting to an overall relative efficiency enhancement of about $0.1 \%$. Our figure of merit, the current enhancement per area per suns-squared, was measured at $1.3 \times 10^{-4} \mathrm{mAcm}^{-2} \odot{ }^{-2}$. This figure should be enhanced by at least one thousand-fold before significant cell improvements are obtained. Nevertheless, we outlined several approaches towards this end.

In our original device, a $1 \mathrm{~cm}$ cuvette was placed behind the solar cell.[17] This arrangement has several drawbacks. Firstly, about half of the upconverted light is lost as it is emitted away from the solar cell. Secondly, by placing a Lambertian reflector at an optimized distance from the solar cell, wavelengths either side of the peak absorbance of the sensitizer may achieve a longer pathlength through the TTA-UC mixture. Furthermore, the cell itself would benefit from improved light-trapping engendered by such a back reflector. 


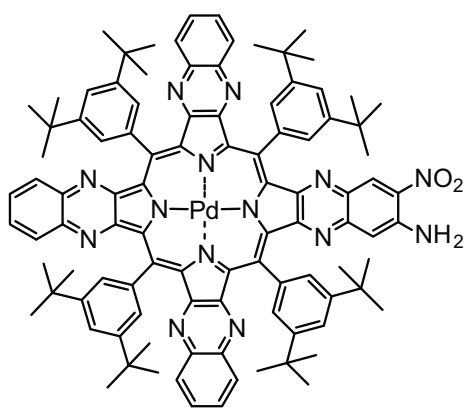

$P Q_{4} P d N A$

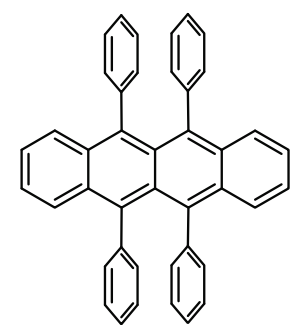

rubrene

FIG. 2. The chemical structures used as sensitizer ( $\left.\mathrm{PdPQ}_{4} \mathrm{NA}\right)$ and emitter (rubrene).

In this contribution, we show that the upconvertor efficiency is roughly doubled with a crude back-scatterer comprised of $100 \mu \mathrm{m}$ diameter silver-coated glass spheres. Furthermore, by increasing the porphyrin concentration, we boost the upconverter by a further factor of two, the combined effects generating a significant improvement as compared to the previous result. We discuss the way forward towards a practical upconvertor for improved solar energy conversion.

\section{EXPERIMENTAL}

Apart from the addition of a backscatterer, and minor details, the experimental conditions were as utilized in our original study. Here we explain the set-up for the convenience of the reader.

\section{A. UC materials}

The upconversion materials used were a subset of the previous study: $\mathrm{PQ}_{4} \mathrm{PdNA}$, as lightharvester (sensitizer); and rubrene as the emitter (Fig.2), dissolved in toluene. Rubrene (Sigma-Aldrich) was used as purchased and the $\mathrm{PQ}_{4} \mathrm{PdNA}$ was prepared in house.[18] Its detailed synthesis and characterisation will be reported elsewhere. The compounds were dissolved in toluene $\left(\mathrm{PQ}_{4} \mathrm{PdNA} 1.2 \times 10^{-3} \mathrm{M}\right.$, rubrene $\left.5.8 \times 10^{-3} \mathrm{M}\right)$ and transferred to a custom made vacuum cuvette $(1 \mathrm{~cm}$ pathlength), in which the solution was degassed by at least 3 freeze-pump-thaw cycles $\left(\sim 10^{-6}\right.$ mbar $)$, thus preventing quenching of the triplet states by molecular oxygen. The front of the UC cuvette was optically coupled to the rear 


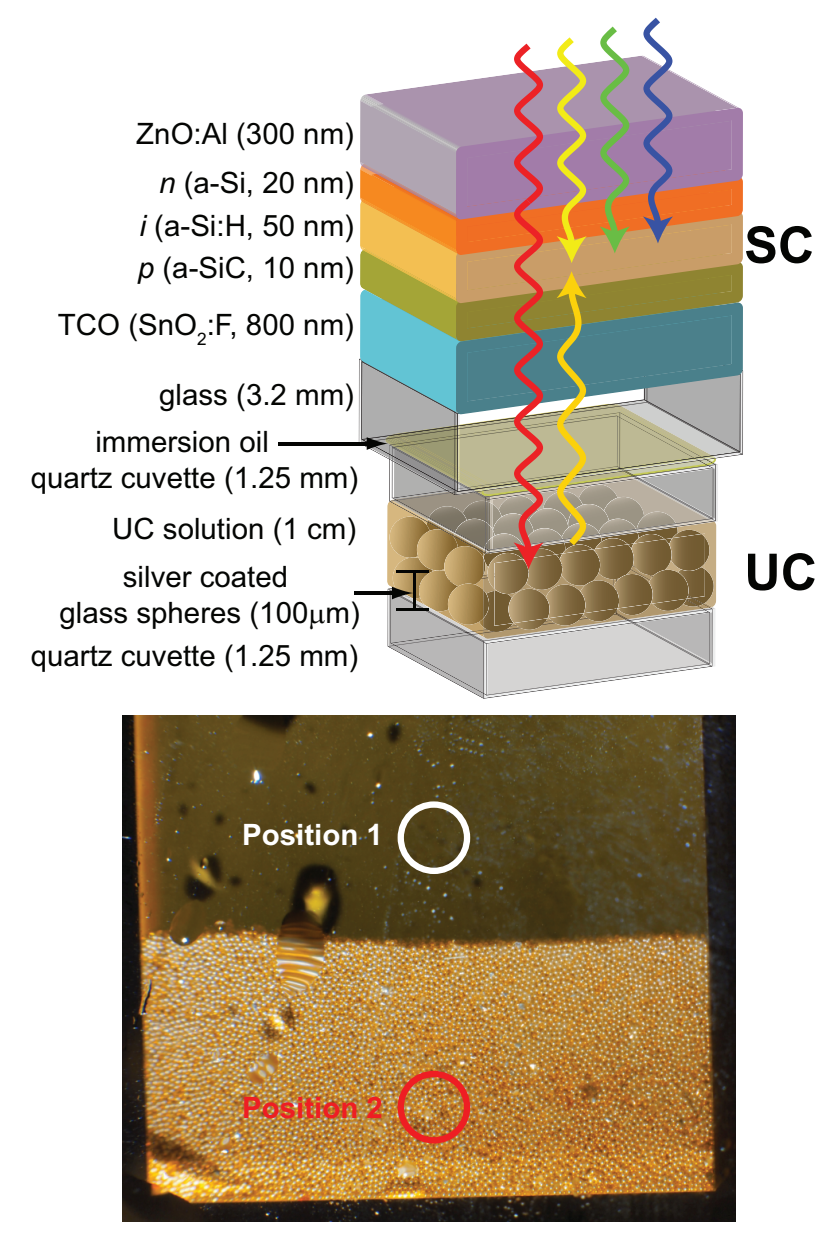

FIG. 3. A cartoon of the device. Low energy (shown in red) photons pass though the $p$ - $i-n$ structure and excite sensitizer molecules in the upconversion unit. Triplet energy is transfered to the emitter molecules which undergo triplet-triplet annihilation, yielding photons of a shorter wavelength (yellow). The $100 \mu \mathrm{m}$ microspheres act as a back-scatter to improve the overall performance.

of the solar cell with a thin film of immersion oil (Sigma-Aldrich, $n_{D}^{20}=1.516$ ).

\section{B. Integrated $\mathrm{SC} / \mathrm{UC}$ device}

The solar cells are identical to our previous study.[17] Thin bifacial a-Si:H $p-i-n$ solar cells were grown at PVcomB at Helmholtz-Zentrum Berlin für Materialien und Energie (HZB). They were produced with a plasma-enhanced chemical vapor deposition (PECVD) cluster tool $(\mathrm{AKT} 1600 \mathrm{~A})$ at $200^{\circ} \mathrm{C}$ on commercial, natively-textured $30 \mathrm{~cm} \times 30 \mathrm{~cm}$ transparent conducting oxide (TCO: $\mathrm{SnO}_{2}: \mathrm{F}$ ) glass substrates. To form bifacial SCs, a $300 \mathrm{~nm}$ film of sputtered ZnO:Al was used as back contact. The highly doped $p$-a-SiC:H and $n$-a-Si:H layers 
were 10 and $20 \mathrm{~nm}$ thick, respectively. To match the absorption profile of the UC unit, the $i$ layer thickness of the $p$ - $i$-n cells was reduced to $100 \mathrm{~nm}$, and we forewent the implementation of light trapping schemes. The superstrate solar cell was illuminated through the transparent ZnO:Al back contact. Bearing in mind the non-ideality of the cell, the AM1.5G efficiency of the cell amounts to $(2 \pm 0.2 \%)$.

The UC unit is placed behind the bifacial a-Si:H SC so that low energy light (below the bandgap) can pass through the SC and reach the UC unit with the upconverted light fluorescing back onto the SC. In order to introduce the scattering surface predicted to improve the performance of the upconvertor, we filled the bottom $5 \mathrm{~mm}$ of the cuvette with $100 \mu \mathrm{m}$ silver-coated glass spheres. The effect of the resulting textured surface is to multipass the incoming light to improve absorption, while also improving the out-coupling of upconverted light. With this set-up we were able to measure both the cases with and without the backscatter with other conditions held constant (see Fig. 3).

\section{Determination of SC enhancement through UC}

The conversion of photons into electrical current may be described by the external quantum efficiency (EQE). Thus, an EQE curve is a spectrum of the linear light-to-current conversion. If the EQE curve is known, then the short-circuit current under the AM1.5G spectrum may be calculated. However, the non-linear response of the upconversion unit cannot be measured in this way. At low light levels the linear response of the intrinsically quadratic process is negligible. Even under monochromated concentrated sunlight, the upconvertor performance is diminished by missing wavelengths of light that would otherwise contribute to the photochemical process. As such, the experiment is performed by measuring the linear response under bias - such that the upconvertor is exposed to a continuous illumination equivalent to a known solar concentration factor.

The light sources used were a monochromated broadband $1 \mathrm{~kW}$ Xe long arc lamp (Oriel) as the probe, and a $0.9 \mathrm{~mW} 670 \mathrm{~nm}$ cw laser diode (Lastek) as the pump. The broadband light of the probe was chopped (Thorlabs, $114 \mathrm{~Hz}$ ) and monochromated (Spectral Products CM110, $14 \mathrm{~nm}$ bandpass). The power of the probe light at each wavelength was of the order $50 \mu \mathrm{W}$. To prevent second order diffraction of short wavelengths, a $405 \mathrm{~nm}$ long pass filter (BLP01-405R-25) was used, restricting the valid range to 405-810 nm. The probe intensity 
was monitored using a glass slide (4\% reflectivity) to reflect part of the beam into a power meter (Newport 1936-C) with a wavelength-calibrated silicon photodiode (918 D-UV-OD3). The pump and probe beams were superposed at the front face of the UC solution, with a spot size of about $1 \mathrm{~mm}^{2}$, having passed through the solar cell.

The short circuit current generated by the solar cell is pre-amplified (Stanford Research SR570), monitored by a lock-in amplifier (National Instruments USB-4431), and analysed with in-house LabVIEW software, suppressing the background current created by the laser diode beam (and other potential sources). Compared to the chopper frequency $(\sim 100 \mathrm{~Hz})$, the build-up and decay of steady-state conditions in the UC unit $(\sim 100 \mu \mathrm{s})[14,15,19]$ are negligible.

The red diode laser $(670 \mathrm{~nm})$ is responsible for pumping porphyrin molecules from their ground state to the first excited state at a certain rate. This rate can be matched to that which would be brought about by a certain concentration of the AM1.5G spectrum, as filtered by the solar cell. This concentration factor is the number of "suns", $\odot)$. In order to gauge the effect of upconversion under the given number of suns, we measured the EQE of the combined UC-SC system with the pump and probe beams aligned and misaligned, respectively. This keeps all cell conditions otherwise equal while switching the upconvertor between dark and operating conditions. Since TTA-UC is a quadratic process at low irradiation, the linear response of the UC unit in the dark is zero for the small probe intensities used here.

\section{Calculation of solar concentration}

The rate of excitation of the porphyrins, $k_{\phi}$, by $1 \odot$ is calculated by multiplication of the AM1.5G solar spectrum, $\rho_{\odot}$, in photons $\mathrm{cm}^{-2} \mathrm{~s}^{-1} \mathrm{~nm}^{-1}$ by the transmission of the solar cell, $T_{\mathrm{SC}}$, and integrating the product of this with the absorption cross section, $\sigma(\lambda)$ in $\mathrm{cm}^{2}$,

$$
k_{\phi \odot}=\int \rho_{\odot}(\lambda) T_{\mathrm{SC}}(\lambda) \sigma(\lambda) \mathrm{d} \lambda
$$

We calculate $k_{\phi \odot}=1.9 \mathrm{~s}^{-1}$ for $\mathrm{PQ}_{4} \mathrm{PdNA}(600-750 \mathrm{~nm}, 100 \mathrm{~nm}$ a-Si:H SC). The irradiation $I_{b}$ of the bias in photons per area per time is used to calculate the experimental pump rate, i.e. $k_{\phi b}=\sigma(670 \mathrm{~nm}) T_{\mathrm{SC}}(670 \mathrm{~nm}) I_{b}$. The ratio $k_{\phi b} / k_{\phi \odot}$ gives the effective solar concentration. 


\section{MODEL}

Under inefficient operating (steady-state) conditions, assuming efficient triplet energy transfer, the rate of production of excited states is equal to the rate of their decay,

$$
k_{\phi} N_{S}=k_{1} N_{T}
$$

where $N_{S}$ and $N_{T}$ are respectively the number densities of ground-state singlet sensitizer and triplet emitter. The first order rate constants, $k_{\phi}$ and $k_{1}$, respectively describe the rate of optical excitation of sensitizers, and first order decay of emitters. In an optically thick cuvette, irradiated on one side, these quantities are dependent on $z$, the penetration depth. Since the upconverted light production is proportional to the triplet concentration squared, one can write that

$$
\mathrm{UC}(z) \propto \frac{k_{\phi}(z)^{2} N_{S}^{2}}{k_{1}^{2}} .
$$

Under experimental conditions, $k_{\phi}$ has contributions from the red diode laser bias, and the monochromated probe. Denoting these additive contributions $k_{\phi b}$ and $k_{\phi p}$,

$$
\mathrm{UC}(z) \propto N_{S}^{2}\left(k_{\phi b}(0) \exp \left(-\alpha_{b} z\right)+k_{\phi p}(0) \exp \left(-\alpha_{p} z\right)\right)^{2}
$$

where the constant $k_{1}$ has been omitted, and the quantities $\alpha_{b}$ and $\alpha_{p}$ denote the absorption coefficients, proportional to the absorption cross sections,

$$
\begin{gathered}
\alpha_{b}=\sigma_{b} N_{S} \\
\alpha_{p}=\sigma_{p} N_{S},
\end{gathered}
$$

at the wavelengths of the bias and the probe, respectively. Integration over $z$ gives

$$
\begin{aligned}
\int d z \mathrm{UC}(z) & \propto N_{S}^{2}\left(\frac{k_{\phi b}^{2}}{2 \alpha_{b}}+\frac{k_{\phi p}^{2}}{2 \alpha_{p}}+\frac{2 k_{\phi p} k_{\phi b}}{\alpha_{p}+\alpha_{b}}\right) \\
& \propto N_{S}\left(\frac{I_{b}^{2} \sigma_{b}}{2}+\frac{I_{p}^{2} \sigma_{p}}{2}+\frac{2 I_{p} I_{b} \sigma_{p} \sigma_{b}}{\sigma_{p}+\sigma_{b}}\right) .
\end{aligned}
$$

Now, the linear response of the upconvertor with probe light is the quantity of interest. The intensity of the probe impinging on the solar cell is larger than $I_{p}$ by a factor $1 / T_{p}$, where $T_{p}$ is the solar cell trasmission at the probed wavelength. Thus

$$
\frac{d \int d z \mathrm{UC}(z)}{d I_{p}(\text { front })} \propto N_{S} T_{p}\left(I_{p} \sigma_{p}+\frac{2 I_{b} \sigma_{p} \sigma_{b}}{\sigma_{p}+\sigma_{b}}\right) .
$$




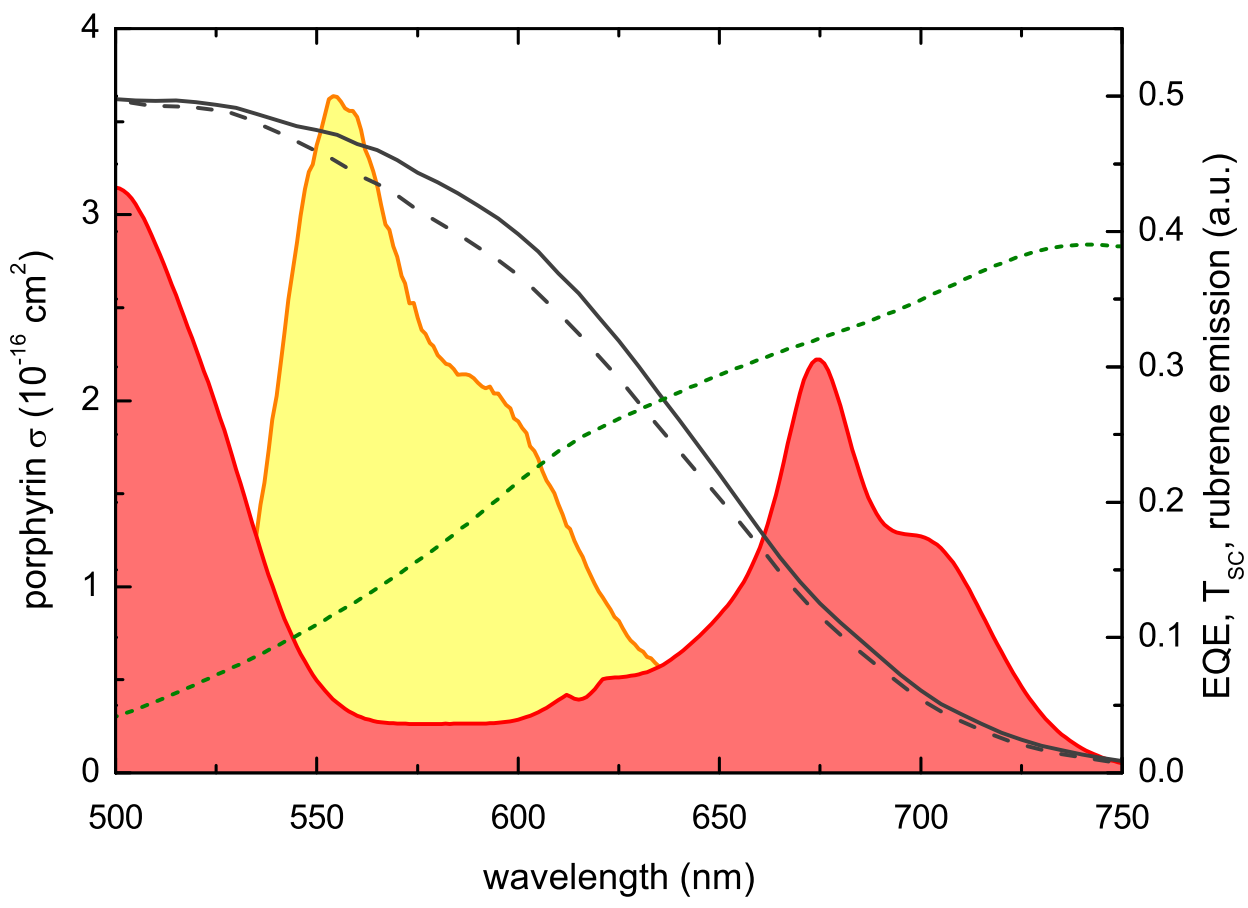

FIG. 4. Red: absorption spectrum of $\mathrm{PdPQ}_{4} \mathrm{NA}$. Yellow: emission spectrum of rubene. Black: EQE curves of the device with (solid) and without (dashed) the back-scattering microspheres. Green: The transmission spectrum of the solar cell.

Since the probe light is indeed small compared to the bias, the first term inside the parentheses can be ignored, and the augmentation of the EQE curve is proportional to the second term. Denoting the EQE in the absence of upconversion $\mathrm{EQE}_{0}$,

$$
\begin{gathered}
\mathrm{EQE}_{\mathrm{UC}}=\mathrm{EQE}_{0}+\text { const. } \times \frac{T_{p} \sigma_{p} \sigma_{b}}{\sigma_{p}+\sigma_{b}} \\
\frac{\mathrm{EQE}_{\mathrm{UC}}}{\mathrm{EQE}_{0}}=1+\text { const. } \times \frac{T_{p}}{\mathrm{EQE}_{0}} \frac{\sigma_{p} \sigma_{b}}{\sigma_{p}+\sigma_{b}} .
\end{gathered}
$$

As such, the observed EQE ratio can be modelled using the absorption spectrum of the porphyrin, and the transmission and EQE curves of the solar cell.

\section{RESULTS AND DISCUSSION}

The EQEs of the solar cell with and without the back-scattering microspheres are displayed in Fig. 4. Both curves drop considerably to the red, indicating that the cells should 
benefit from upconversion of photons in the $700 \mathrm{~nm}$ region into the $550 \mathrm{~nm}$ region. Indeed, as can be seen, the transmission of photons in the $700 \mathrm{~nm}$ region would allow these photons to be unconverted at the rear of the solar cell. Even without upconversion, it is clear that the the addition of silver-coated microspheres to the upconversion cuvette increases the EQE of the solar cell. This is due to a light-trapping effect, whereby transmitted photons reflected at oblique angles re-enter the solar cell such that they trace out a longer path length inside the active layer. Integrated over the AM1.5G spectrum, the increased EQE amounts to a short circuit current of $6.895 \mathrm{~mA}$, a $4 \%$ increase compared to the device without the silver microspheres.

The ratio of EQE curves, with and without upconversion, is shown in Fig. 5 for the cases with and without the reflecting spheres. The control experiment (no back reflector, position 1) shows a $1 \%$ increase in the EQE curve in the $700-750 \mathrm{~nm}$ region, obtained at an equivalent solar concentration of $24 \odot$. This is roughly the same increase as previously reported for this porphyrin, but under half the solar concentration. This enhancement is due to the higher porphyrin concentration used presently. Repeating the experiment lower on the cuvette at position 2, with the reflective microspheres, doubles the enhancement due to upconversion. We can calculate the total short circuit current density increase of the solar cell due to UC, under the equivalent solar concentration as

$$
\Delta J_{\mathrm{SC}}^{\mathrm{UC}}=e \int\left(\mathrm{EQE}_{\mathrm{UC}}-\mathrm{EQE}_{0}\right)(\lambda) f_{c} \rho_{\odot}(\lambda) d \lambda
$$

where $e$ is the elementary charge, $f_{c}$ the concentration factor, $\rho(\lambda)$ is the AM1.5 solar flux in photons per area per time per wavelength. Under the experimental conditions we obtain $\Delta J_{\mathrm{SC}}^{\mathrm{UC}}=0.096 \mathrm{~mA} \mathrm{~cm}^{-2}$ and $\Delta J_{\mathrm{SC}}^{\mathrm{UC}}=0.281 \mathrm{~mA} \mathrm{~cm}^{-2}$, for the upconvertors with and without the microspheres, respectively, having integrated over the raw EQEs. The figure of merit proposed for our device is normalized for the solar concentration squared, yielding $\zeta=1.7 \times 10^{-4} \mathrm{~mA} \mathrm{~cm}^{-2} \odot^{-2}$ and $4.9 \times 10^{-4} \mathrm{~mA} \mathrm{~cm}^{-2} \odot^{-2}$. As such, it would appear that the effect of the back-scattering layer is to improve the upconvertor by a factor of three. However, the noise in the data, especially at wavelengths $>720 \mathrm{~nm}$ where the EQE is very small, does indicate a level of uncertainty. The solid lines in Fig. 5 are singleparameter fits using the model outlined above. As can be seen, the form of the curve is more than satisfactory. The EQE enhancement peaks at a higher wavelength than the sensitizer absorption spectrum due to two effects. Firstly, the transmission spectrum of the cell is rising 


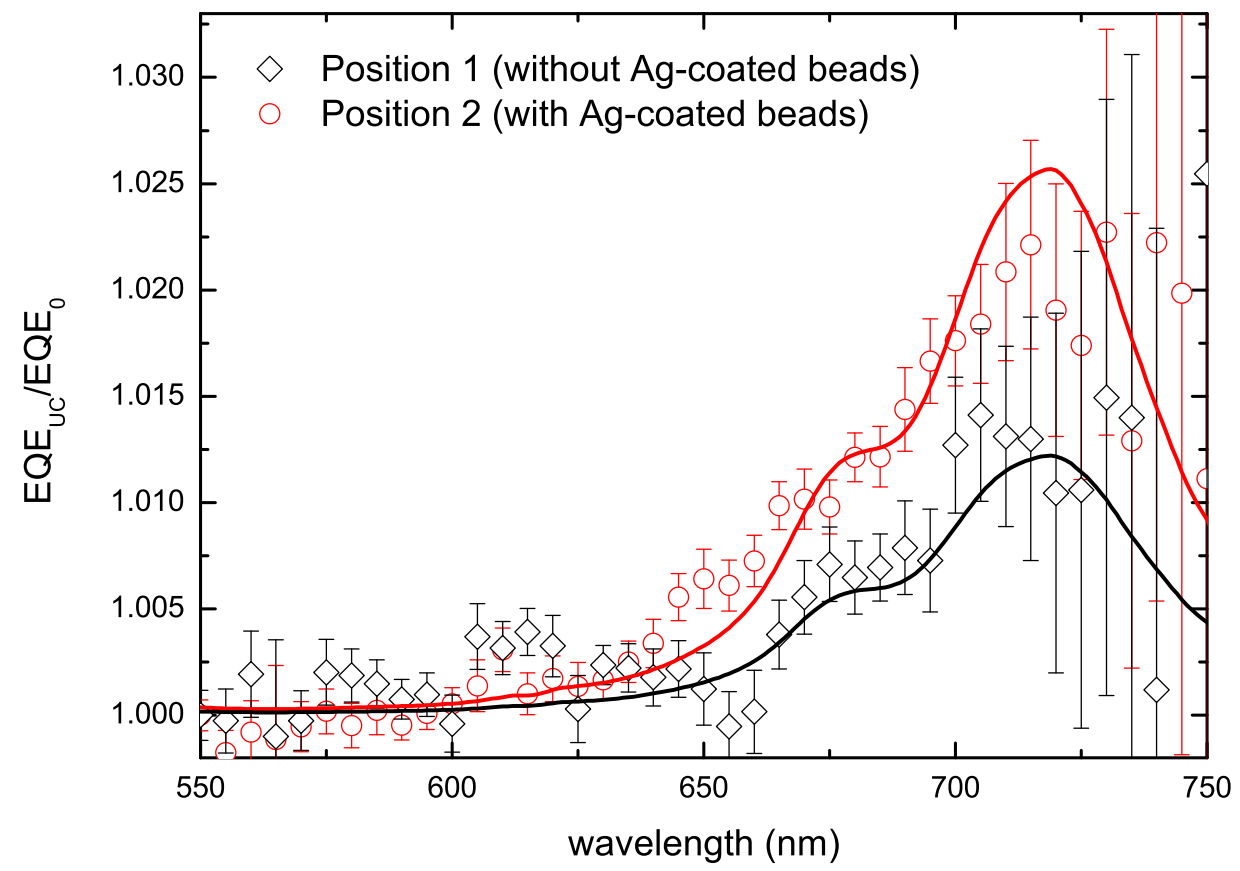

FIG. 5. Effect of UC unit on the solar cell performance from the ratio of SC response curves. The straight lines display the expected spectral shape (see text).

to longer wavelengths, and thus longer wavelengths are able to penetrate into the upconvertor more effectively. Secondly, since the plotted result is a relative EQE enhancement, this is naturally higher where the intrinsic EQE is diminished, which is to longer wavelengths. Consequently, the form of Eq. 11 predicts the observed peak at $\sim 720 \mathrm{~nm}$. Now, by using the fitted EQE enhancement and repeating the calculations, we obtain $\Delta J_{\mathrm{SC}}^{\mathrm{UC}}=0.097 \mathrm{~mA} \mathrm{~cm}^{-2}$ and $\Delta J_{\mathrm{SC}}^{\mathrm{UC}}=0.204 \mathrm{~mA} \mathrm{~cm}^{-2}$, which represents a little more than a doubling of the short circuit current due to upconversion, in the presence of a back-scatterer.

In our previous work,[17] we predicted that an ideal Lambertian back-scattering layer would enhance the upconvertor by a factor of 3.6. A simple specular reflector (mirror) would improve the device by a factor of 2.6. This is due to two factors. Firstly, the upconverted light is better coupled into the solar cell. As the rubrene fluorescence is necessarily isotropic, without a rear reflector, one would expect half of the upconverted light to be lost, although a portion is self absorbed and re-emitted. As such, a naive expectation is a doubling of upconverted light entering the cell with a mirror placed at a judicious distance. This distance 
was calculated to scale as $d=1 / \alpha_{\text {peak }}$. At this distance, the light at the peak absorbance of the sensitizer is attenuated to $1 / e$ on the first pass to the mirror, and upon retroreflection is diminished to $1 / e^{2}$ of its original intensity as it reaches the front of the cuvette. However, if the rear reflector is Lambertian, rather than specular, then much longer pathlengths are possible, with more complete light absorption achieved.

The present reflector design is far from ideal, so the observed improvement of at least a factor of two is encouraging. We selected $100 \mu \mathrm{m}$ silver spheres to provide the appropriate length-scale. Operating under a concentration of $1.2 \mathrm{mM}$, with a molar extinction coefficient of about $55000 \mathrm{M}^{-1} \mathrm{~cm}^{-1}, 1 / \alpha=150 \mu \mathrm{m}$. With a close-packed structure of $100 \mu \mathrm{m}$ spheres, most of the surface area presents an optical depth less than this. Nevertheless, most of the light rays impinging on the spheres are reflected at oblique angles. The solution to the raytracing equations required to model this device are complicated, yielding fractal patterns of reflections (see Ref. 20). The region between three spheres represents a so-called fractal vortex, with multiple reflections ensuring complete light absorption. The remainder of the illuminated volume can out-couple the upconverted light in at most one refection. Nevertheless, despite some interesting characteristics, the microsphere back-scatterer is sub-optimal, and we believe that the stated 3.6-fold improvement can be obtained with a Lambertian surface at the appropriate distance.

\section{CONCLUDING REMARKS}

Figure 6 shows the evolution of our figure of merit for upconversion applied to second generation solar cells. Our first report generated a figure of $1.3 \times 10^{-4} \mathrm{~mA} \mathrm{~cm}^{-2} \odot^{-2}$, which was already 200 times that obtained with rare-earth ions. Here we obtain a conservative value of $3.5 \times 10^{-4} \mathrm{~mA} \mathrm{~cm}^{-2} \odot^{-2}$, which is 2.7 times the previous result. About a factor of two can be attributed to the back reflector, and the remainder is due to a higher porphyrin concentration. The result is still about two-three orders of magnitude lower than required to generate significant solar cell enhancements. Nevertheless, there are several improvements to be made. Firstly, the back-scatter needs to be optimized. This will improve the device by another $50 \%$. Secondly, plasmonic field enhancement may be used to concentrate light absorption and thus increase local triplet concentrations. Lastly, if the light harvesting species can be concentrated without undue quenching of excited states, then the upconverted 


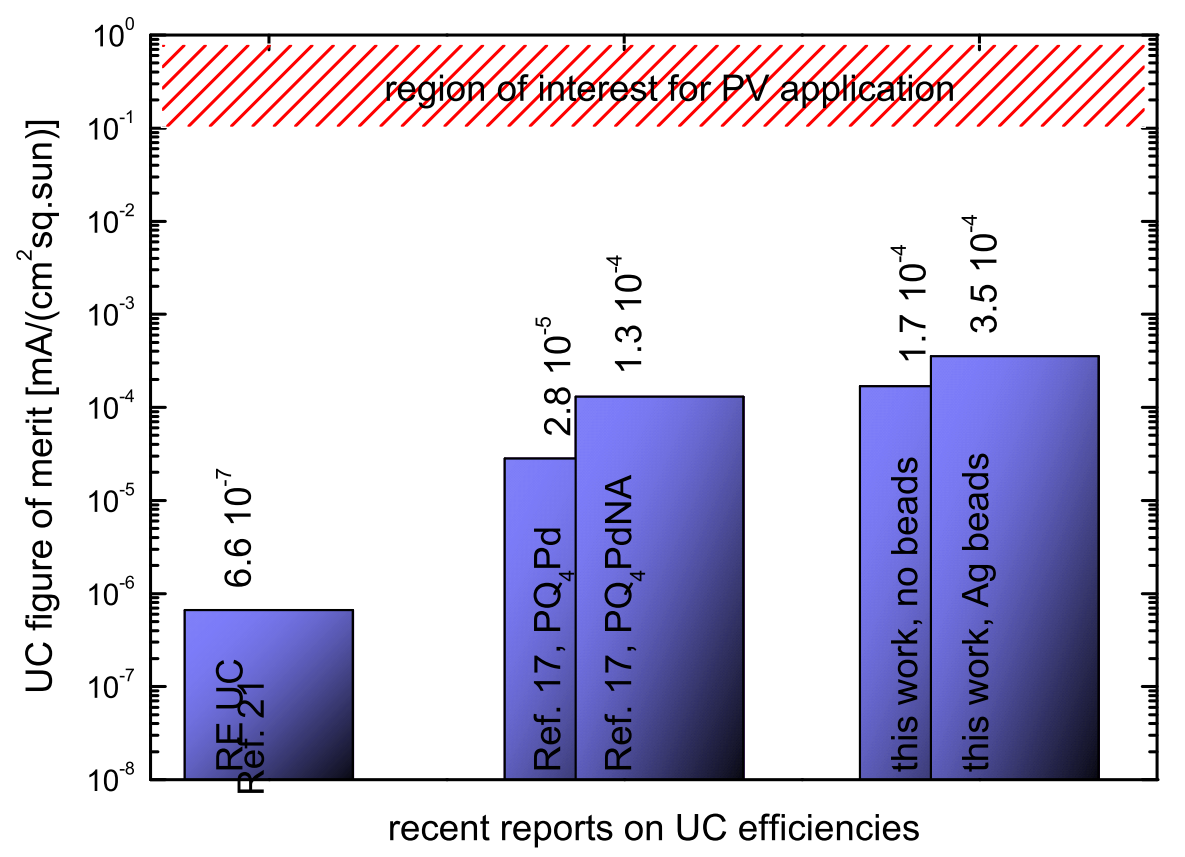

FIG. 6. The evolution of the figure of merit for upconversion applied to amorphous silicon solar cells. Our original publication (Ref. 17) outperformed rare-earths (Ref. 21) by 200 times. Addition of a back-scatterer and increasing the concentration (this work) improves this by a further factor of 3 .

light output can be commensurately increased. This might be achieved with a self-assembled material - a subject currently under investigation in our group.

\section{ACKNOWLEDGEMENTS}

Y.C. acknowledges The University of Sydney for a Henry, Bertie and Florence Mabel Gritton Scholarship. B.F. and T.F.S. acknowledge the Alexander von Humboldt-Foundation for respective Feodor Lynen fellowships. R.G.C.R.C. acknowledges the Australian Solar Institute for a postdoctoral research fellowship. K.L. is indebted to the Deutsche Forschungsgemeinschaft (DFG) for grant 583727 which initiated this German-Australian bilateral cooperation. We thank A. Stanco and Lastek for the gift of the $670 \mathrm{~nm}$ diode laser, M. Kaegi for making the SC holder, and T. Hänel (HZB) for EQE measurements. This research project 
is funded by the Australian Solar Institute, with contributions from The New South Wales Government and The University of Sydney. Aspects of this research was supported under Australian Research Council's Discovery Projects funding scheme (DP110103300). Equipment was purchased with support from the Australian Research Council (LE0668257). The work related tot the SC preparation at HZB was funded by the Federal Ministry of Education and Research (BMBF) and the state government of Berlin (SENBWF) in the framework of the program "Spitzenforschung und Innovation in den Neuen Ländern" (03IS2151).

[1] R. E. Smalley, MRS Bulletin 30, 412 (2005).

[2] N. S. Lewis, Science 315, 798 (2007).

[3] W. Shockley and H. J. Queisser, Journal of Applied Physics 32, 510 (1961).

[4] M. A. Green, Third Generation Photovoltaics: Advances Solar Energy Conversion (SpringerVerlag, Heidelberg, 2003).

[5] M. A. Green, Progress in Photovoltaics 17, 183 (2009).

[6] M. Grätzel, Nature 414 (2001).

[7] G. Li, V. Shrotriya, J. S. Huang, Y. Yao, T. Moriarty, K. Emery, and Y. Yang, Nature Mater. 4 (2005).

[8] A. Yella, H.-W. Lee, H. N. Tsao, C. Yi, A. K. Chandiran, M. Khaja Nazeeruddin, E. W.-G. Diau, C.-Y. Yeh, , S. M. Zakeeruddin, and M. Graetzel, Science 334, 629 (2011).

[9] M. C. Hanna and A. J. Nozik, Journal of Applied Physics 100 (2006).

[10] L. C. Hirst and N. J. Ekins-Daukes, Progress in Photovoltaics 19, 286 (2011).

[11] T. Trupke, M. A. Green, and P. Würfel, J. Appl. Phys. 92, 4117 (2002).

[12] T. N. Singh-Rachford and F. N. Castellano, Coordination Chemistry Reviews 254, 2560 (2010).

[13] T. N. Singh-Rachford, A. Haefele, R. Ziessel, and F. N. Castellano, J. Am. Chem. Soc. 130, 16164 (2008).

[14] Y. Cheng, T. Khoury, R. G. C. R. Clady, M. J. Y. Tayebjee, N. J. Ekins-Daukes, M. J. Crossley, and T. W. Schmidt, Phys. Chem. Chem. Phys. 12, 66 (2010).

[15] Y. Cheng, B. Fückel, T. Khoury, R. G. C. R. Clady, M. J. Y. Tayebjee, N. J. Ekins-Daukes, M. J. Crossley, and T. W. Schmidt, J. Phys. Chem. Lett. 1, 1795 (2010). 
[16] R. S. Khnayzer, J. Blumhoff, J. A. Harrington, A. Haefele, F. Deng, and F. N. Castellano, Chem. Commun. 48, 209 (2012).

[17] Y. Y. Cheng, B. Fückel, R. W. MacQueen, T. Khoury, R. G. C. R. Clady, T. F. Schulze, N. J. Ekins-Daukes, M. J. Crossley, B. Stannowski, K. Lips, and T. W. Schmidt, Energy Environ. Sci. (2012), 10.1039/C2EE21136J, accepted manuscript.

[18] T. Khoury and M. J. Crossley, Chem. Commun. 46, 4851 (2007).

[19] T. N. Singh-Rachford and F. N. Castellano, Inorg. Chem. 48, 2541 (2009).

[20] D. Sweet, E. Ott, and J. A. Yorke, Nature 399 (1999).

[21] J. de Wild, J. K. Rath, A. Meijerink, W. G. J. H. M. van Sark, and R. E. I. Schropp, Solar Energy Materials and Solar Cells 94, 2395 (2010). 